\title{
An Unlikely Combination of Experiments with a Novel High-Voltage CIGS Photovoltaic Array
}

J.A. del Cueto, B.R. Sekulic

National Renewable Energy Laboratory

Golden, Colorado

United States of America

$4^{\text {th }}$ World Conference on Photovoltaic Energy Conversion Waikoloa, Hawaii

$$
\text { May 7-12, } 2006
$$




\section{Disclaimer and Government License}

This work has been authored by Midwest Research Institute (MRI) under Contract No. DE-AC36-99GO10337 with the U.S. Department of Energy (the "DOE"). The United States Government (the "Government") retains and the publisher, by accepting the work for publication, acknowledges that the Government retains a non-exclusive, paid-up, irrevocable, worldwide license to publish or reproduce the published form of this work, or allow others to do so, for Government purposes.

Neither MRI, the DOE, the Government, nor any other agency thereof, nor any of their employees, makes any warranty, express or implied, or assumes any liability or responsibility for the accuracy, completeness, or usefulness of any information, apparatus, product, or process disclosed, or represents that its use would not infringe any privately owned rights. Reference herein to any specific commercial product, process, or service by trade name, trademark, manufacturer, or otherwise does not constitute or imply its endorsement, recommendation, or favoring by the Government or any agency thereof. The views and opinions of the authors and/or presenters expressed herein do not necessarily state or reflect those of MRI, the DOE, the Government, or any agency thereof. 


\section{Introduction}

a Long-term (2020) goal of the US Solar Program Multi-Year Plan:

$>$ Commercialization of photovoltaic (PV) modules with 30-year lifetimes or more, capable of sustaining less than $0.5 \%$ annual performance degradation rate, and at costs consistent with market-rates of electricity.

- Motivation: quantify the performance, stability and reliability of high efficiency thin-film copperindium-diselenide (CIS) PV modules in a highvoltage array. 


\section{Motivation: PV Module Reliability}

- High voltage is a known stress-mechanism that precipitates PV module degradation:

$>$ In the 1980's, the Jet Propulsion Laboratory (JPL) investigated the connection between high-voltage leakage currents from modules and their degradation:

$>$ Found that series resistance increases, brought about by electrochemical corrosion of contacts, is a prime failure mechanism induced by high-voltage stress

$>$ Established key thresholds of accumulated charge that would result in 50\% failure in certain PV modules:

* For crystalline-silicon (c-Si) ranging 1-10 coulombs per linear centimeter $(\mathrm{C} / \mathrm{cm})$ of module perimeter,

* For amorphous-silicon (a-Si) modules $0.1-1 \mathrm{C} / \mathrm{cm}$ 


\section{Motivation: PV Performance \& Stability}

- Actual energy production of modules under real field conditions continues to be an issue:

$>$ Currently only measurements of power at Standard Reporting Conditions (SRC) are used to rate modules

* But SRC misses most of actual operating conditions

- PV arrays commonly employ inverters and measurements reported usually include only optimum power-point voltage, current and power

$>$ When performance degrades, measurements of optimum power point voltage \& current are usually not enough to discern the failure mode,

$>$ Would benefit to have more in-depth I-V characterization. 


\section{Goals of This Study}

$\checkmark$ Parameterize current-voltage (I-V) performance over a wide range of illumination \& temperatures:

$>50-1150 \mathrm{~W} / \mathrm{m}^{2}$ irradiance, $5^{\circ}-65^{\circ} \mathrm{C}$

$>$ Obtain array temperature coefficients

$>$ Quantify energy production

$\checkmark$ Investigate high-voltage leakage currents from the CIS modules in a high-voltage array:

$>$ Determine dependence on moisture, temperature, and voltage bias

$>$ Ascertain corrosion problems if any

๑ Study long-term power \& energy production stability. 


\section{HVST2 CIGS Array}

\section{a 24 Shell Solar thin-film CIGS modules}

$>$ Nominally $\pm 300 \mathrm{VDC}$ open-circuit \& $1 \mathrm{~kW}$ total array power

$>$ Deployed in 2 bipolar strings, 12 modules in each connected in series

$>$ Each string: aperture area $4.877 \mathrm{~m}^{2}$, segregated into 2 groups of 6 modules each group for each string

$>$ Each group of 6 modules perimeter length $\sim 1.915 \mathrm{~m}$

$>$ Efficiency baseline tested at standard test conditions (STC, $\eta_{S T C}$ ) prior to deployment:

* All modules tested between 36 and $44 \mathrm{~W}$ power, at STC,

- Average $\eta_{S T C}$ for the positive (+) and negative (-) strings of the array: respectively, $9.64 \%$ and $9.53 \%$.

$>$ Dry hi-pot and wet hi-pot tests : for both tests, the leakage currents ranged 0.1-0.6 microamps. 


\section{HVST2 CIGS Array}

\section{Outdoor Test Facility (OTF)}

View Looking East Toward Array

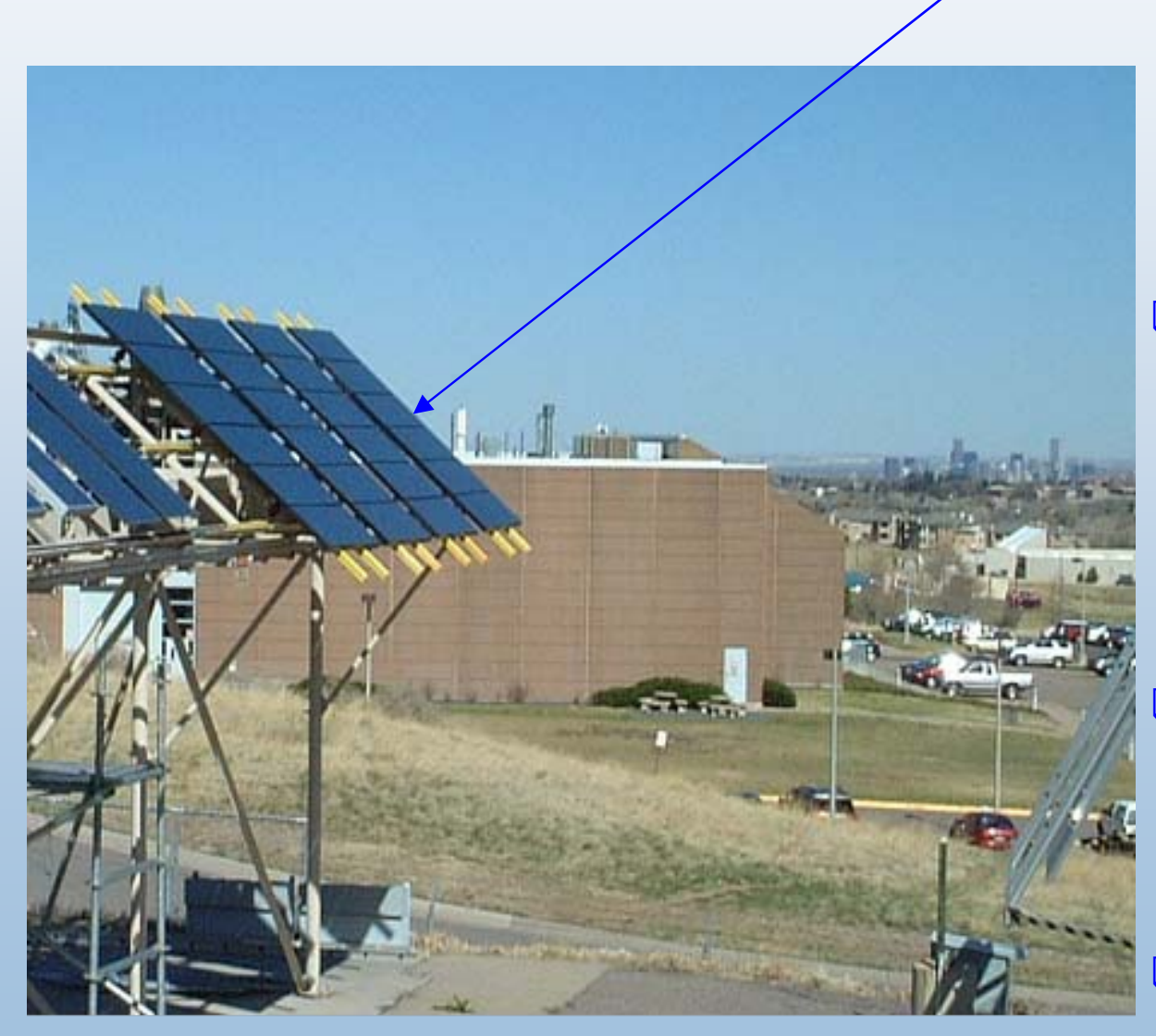

- Current-Voltage (I-V) control $\&$ measurements:

$>$ via programmable e-load

$>\mathrm{I}-\mathrm{V}$ traces once every $15 \mathrm{~min}$, in $1^{\text {st }}$ quadrant

$>$ peak-power other times

- Temperatures:

$>$ type ' $\mathrm{T}$ ' TC back-of-module one for each $+/$ - string $>$ Air Temp. \& humidity (RH)

- Modules mounted facing south, at $\sim 40^{\circ}$ tilt $\sim$ latitude $\sim 39.7^{\circ}$, plane-of-array (POA) Irradiance sensed with pyranometers, same POA 


\section{HVST2 Array Connection}

High Voltage Array: 24 CIGS PV modules 2 strings $+/$ - , with 12 modules per string, 2 groups of 6 modules in series each, \pm 300 volts Voc , 2.5 amps Isc from each string

- String + String

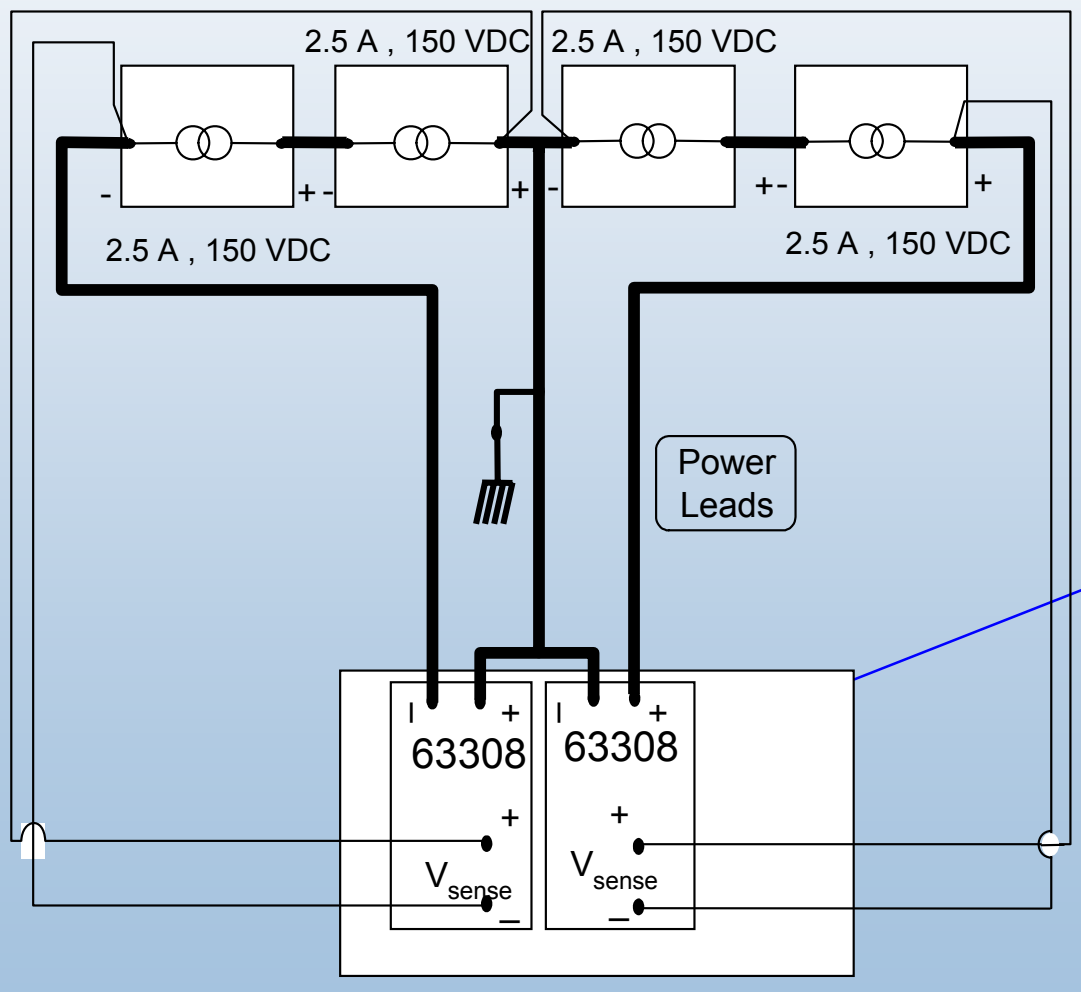

Programmable Electronic Load: Control Modes: constant current or voltage, resistance Loading
24 CIGS modules in positive $(+)$ and negative (-) strings

$>12$ modules per string, 2 groups of 6 modules each per string

> Frames not grounded at supports, but instead connected to resistive network and then to ground to facilitate leakage current sensing

- Electrical Characterization \& Control

> via dual-channel Programmable Electronic Load (PEL) interfaced to Data Acquisition System (DAS) running Visual BASIC Code

> PEL is not a power supply/source

> PEL can control in constant voltage, current or resistance

$>$ DAS sends varying set-points to PEL to trace I-V curves or track peak power 


\section{HVST2 Array Leakage Currents Sensing}

- Frames allowed to float and electrically daisy-chained together on support structure

- Electrical connection made to resistive network before ground:

> Leakage currents pass through $52.5 \mathrm{kOhm}$ resistor combination,

> Leakage currents appear as voltage across $2.49 \mathrm{kOhm}$ resistor, are sensed by CR23x data-logger differential input channels

$>$ Arc by-pass to ground provided in case of too much leakage.

- Each group of 6 modules has own sensing circuit

$>$ Group 1 always higher offset bias being further from ground

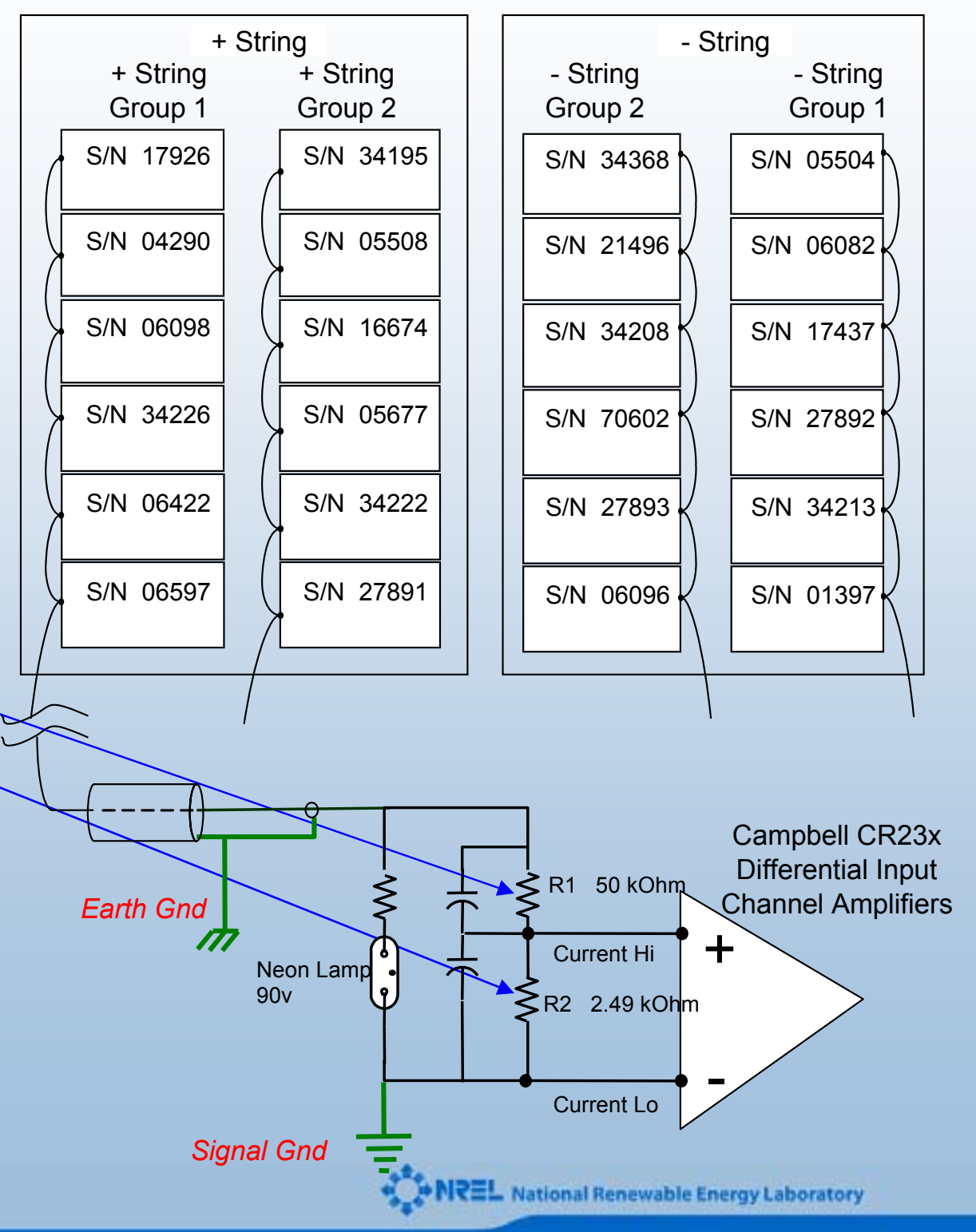




\section{Data Analysis}

1. Integration of peak-power tracking and irradiance data versus time yielding daily energy output, insolation

2. Accumulation of all I-V trace records, statistical filtering for predominantly clear-sky conditions, followed by segregation into irradiance bins \& regression vs. Temp.

3. Integration of the leakage current data through module frames resulting in accumulated daily leakage charge.

4. Efficiency quotients from two distinct angles:

i. usual I-V trace data representing power measurements from each string, divided by the incident power on each string,

ii. Effective Efficiency $\left(\eta_{E F F}\right)=$ ratio of the daily energy output from each string divided by the daily insolation energy

$$
\eta_{E F F}=\int_{\text {Daily }} P_{M A X} \cdot d t /\left[\text { Area }_{\text {String }} \cdot \int_{\text {Daily }} \text { Irr } \cdot d t\right]
$$




\section{Peak Power Tracking Data}

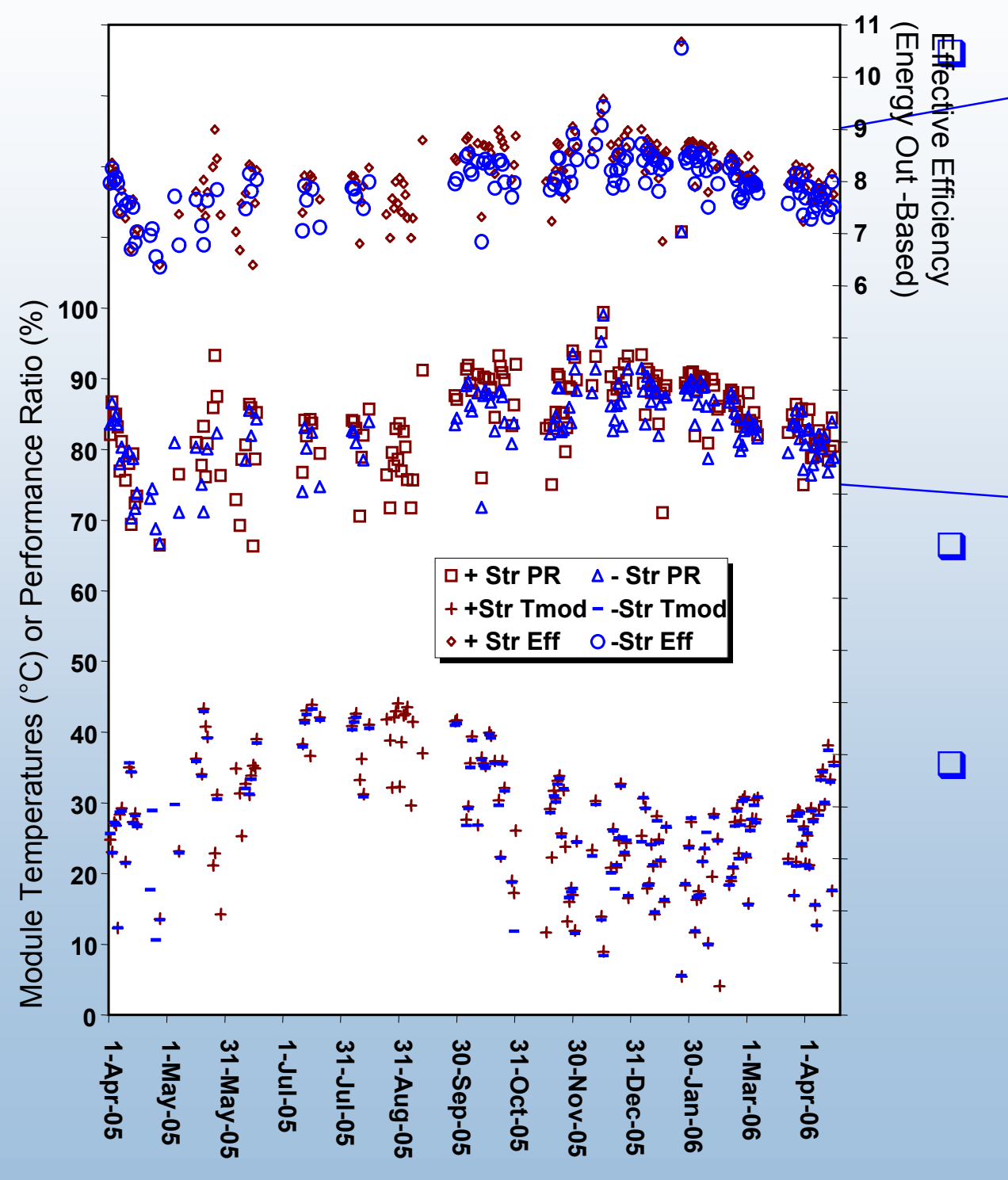

Top: Effective efficiency $\left(\eta_{E F F}\right)$ derived daily from energy output (time-integrated power)

$>$ Not temperature corrected due to integration and convolution of all field conditions occurring daily

> Varies seasonally $\sim 7 \%-9 \frac{1}{2} \%$

口 Mid: Performance ratio (PR)

$>\mathrm{PR}=\eta_{E F F} / \eta_{S T C}$ energy-based, vary $70 \%-95 \%$ of $\eta_{\text {STC }}$

Bottom: +/- strings sampled average daytime module temperatures

$>\eta_{E F F}$ is function of temperature,

$>\left(1 / \eta_{E F F}\right) d \eta_{E F F} / d T_{\text {air }} \sim-0.38 \% /{ }^{\circ} \mathrm{C}$

- $T_{\text {air }} \rightarrow$ average daytime air temp. 


\section{Performance From Full I-V Traces}

- FF (top) and Efficiency (bottom) from full I-V traces, corrected to $25^{\circ} \mathrm{C}$ temperature, for irradiance $=$ $1000 \pm 50 \mathrm{~W} / \mathrm{m}^{2}$ depicted

$>$ No statistically significant changes in efficiency or FF with time in $1^{\text {st }}$ year

$>$ Seasonal variations observed

$>$ + string: $9.1 \% \pm 0.3 \%$ efficiency, $61 \%-62 \% \mathrm{FF}$

$>$ - string: $8.9 \% \pm 0.3 \%$, efficiency, $\sim 60 \% \mathrm{FF}$

a Actual FF determination allows closer scrutiny in event of degradation (e.g., series, shunt resistances).
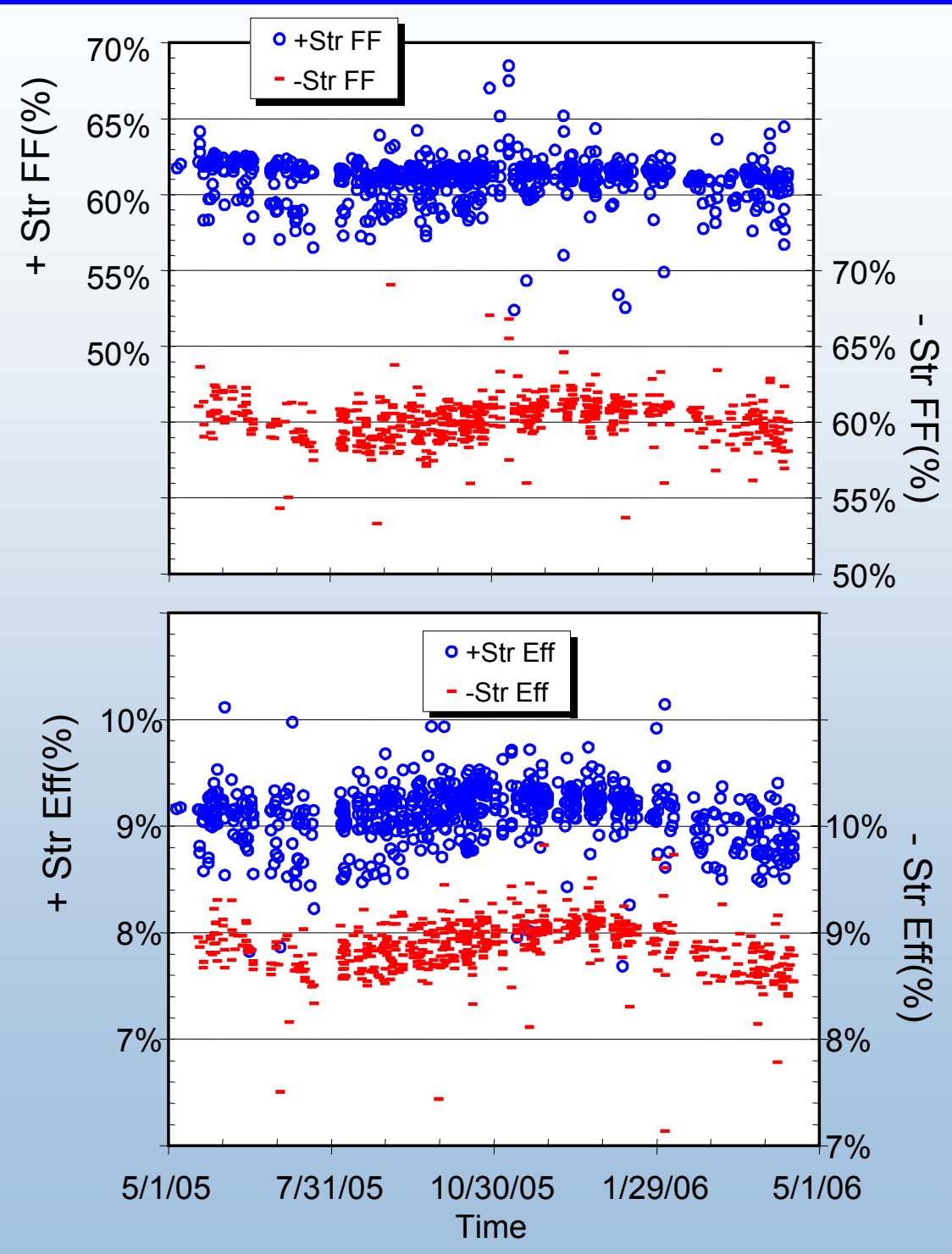


\section{Voc \& FF From Full I-V Traces}

- Dependence analyzed across all irradiance every $50 \mathrm{~W} / \mathrm{m}^{2}$, corrected to $25^{\circ} \mathrm{C}$ module temperature (Tmod)

$>$ Voc shows logarithmic type dependence with irradiance

* Coefficients $(1 / V o c)$ dVoc/dTmod vary $\sim-0.37$ to $-0.30 \% /{ }^{\circ} \mathrm{C}$

$>$ FF data, seem to peak at 300 $\mathrm{W} / \mathrm{m}^{2}$, then decrease with increasing irradiance

* Consistent with series-resistance limited behavior

- Drops off at low irradiance

* Coefficients (1/FF) dFF/dTmod vary $\sim-0.05$ to $+0.20 \% /{ }^{\circ} \mathrm{C}$ going

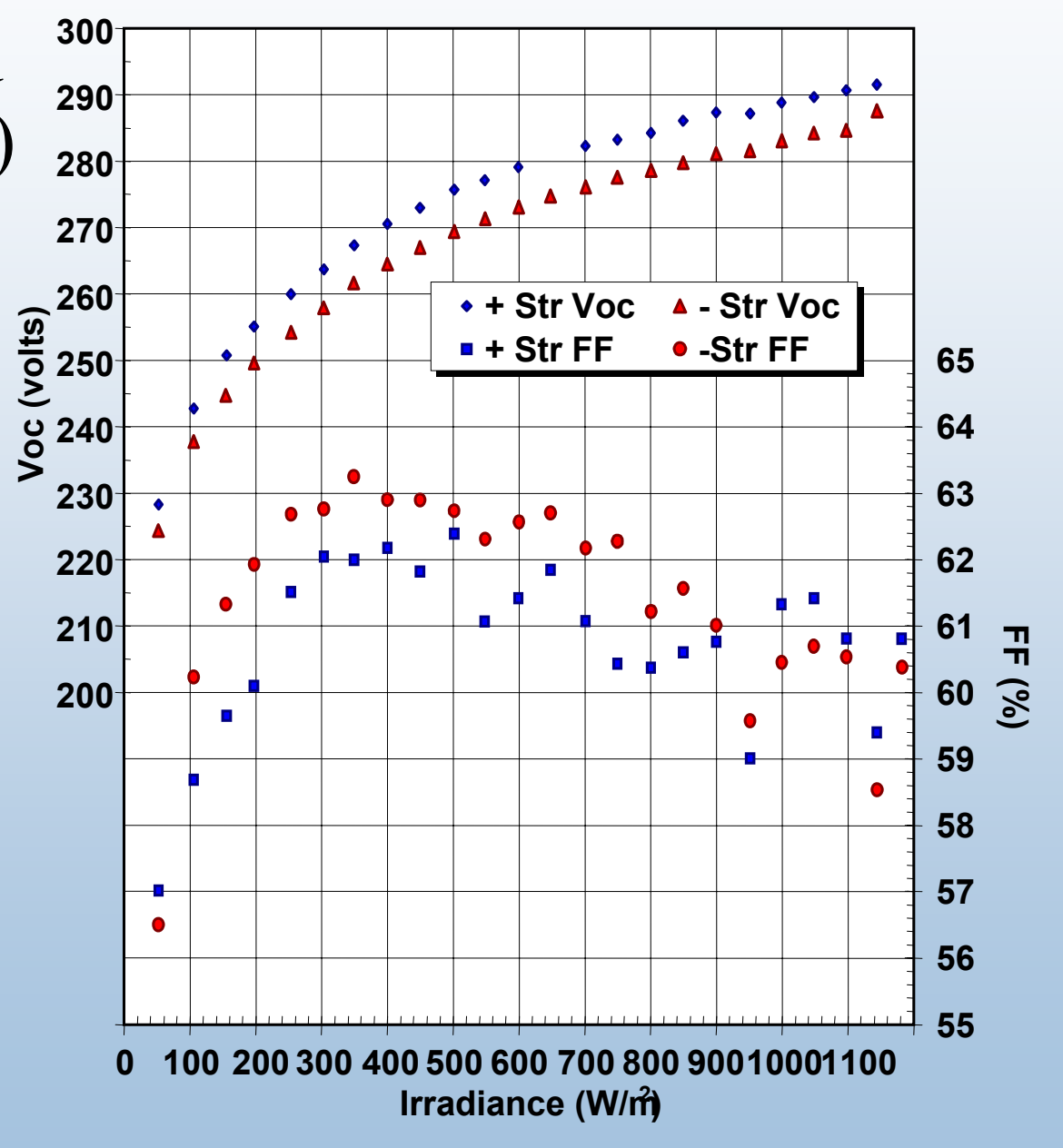
from high to low irradiance 


\section{Leakage Currents}

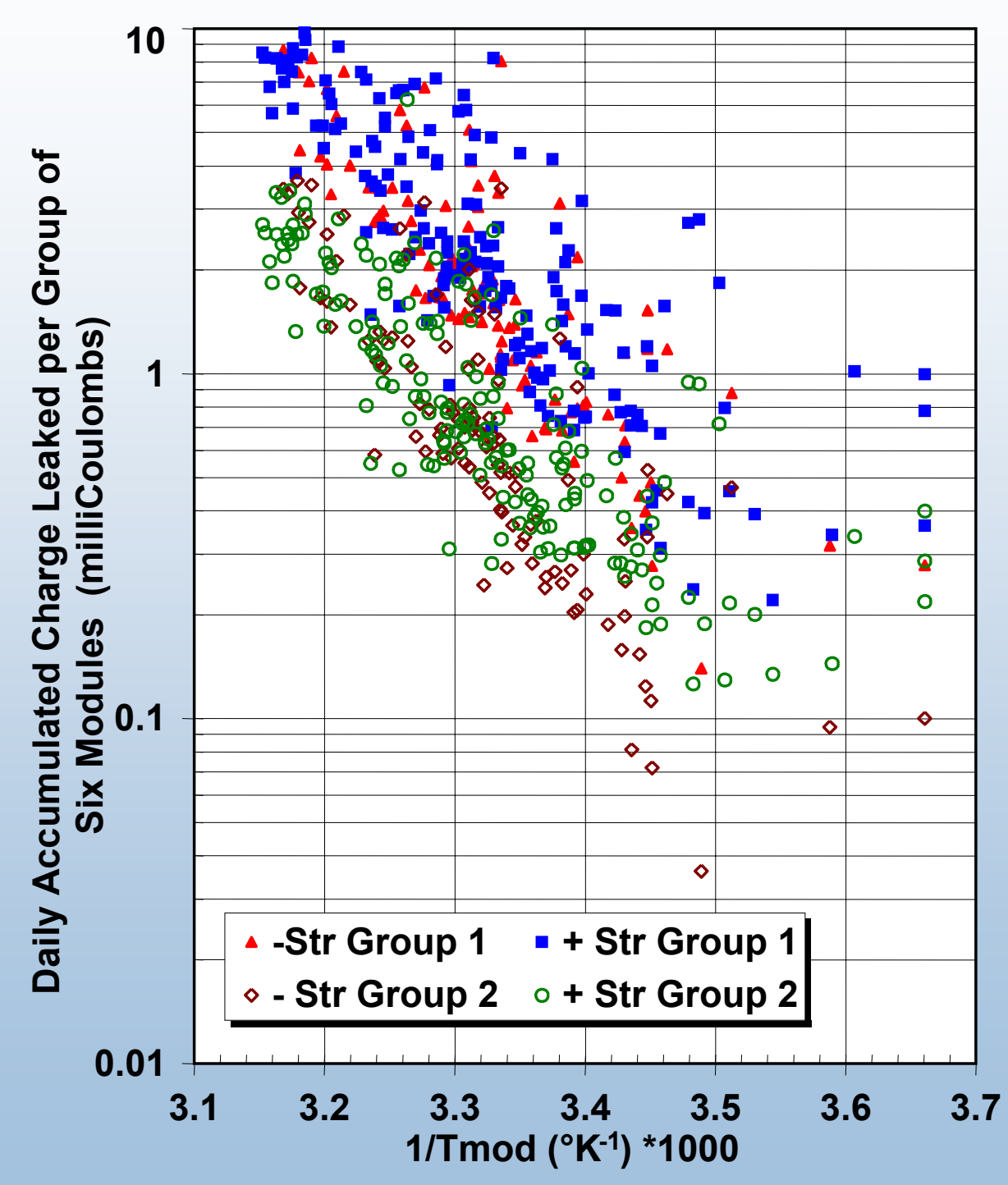

- $\mathrm{Q}_{\mathrm{L}}=$ Daily integrated leakage charge: $Q_{\text {Leak }}=\int I_{\text {Leak }} \cdot d t$

๑ Computed \& plotted for each group of 6 modules vs. average daytime temperatures

- Plotted on Arrhenius Graph

$>$ Appears thermally activated

> Scale 0.01-10 milliCoulombs

口 Variations or scatter:

$>$ Sizes of group-1 $\mathrm{Q}_{\mathrm{L}}$ are $\sim 2.8$ - 2.9 * Group-2 $\mathrm{Q}_{\mathrm{L}}$, reflects partition of voltages between modules \& higher offset bias for group 1

$>$ Humidity, integration over day convolutes multiple humidity values 


\section{Leakage Charge: Activation and Size}

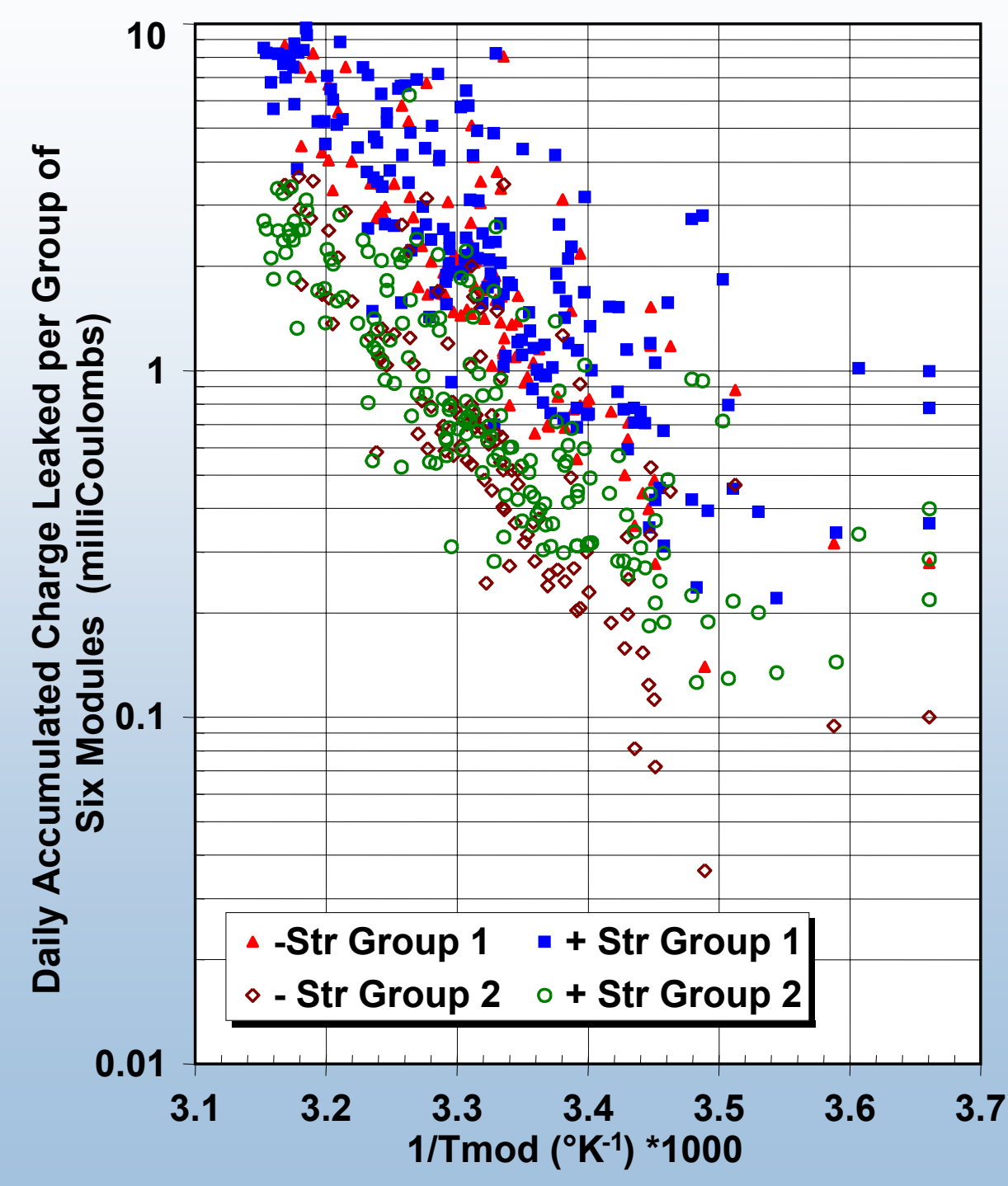

- $\mathrm{Q}_{\mathrm{L}}=$ integrated leakage charge

- Thermal Activation energies:

$>0.6-0.78 \mathrm{eV}$

$>$ consistent with leakage conduction through soda-lime glass

口 For largest leakage $\sim 10 \mathrm{mC}$, Tmod average $\sim 23-30^{\circ} \mathrm{C}$

$>$ Not a problem in Colorado due to our cold dry climate

$>$ In hotter climate, say with 200 days/year at these temperatures,

$*$ In 10 years, would accumulate $\sim 20$

Coulombs leakage for each group of 6 modules or about $0.1 \mathrm{C} / \mathrm{cm}$

$* 0.1 \mathrm{C} / \mathrm{cm}=>$ is right at threshold for $50 \%$ failure rate determined by JPL for a-Si thin-film modules 


\section{Leakage Currents: Response to Array Bias}

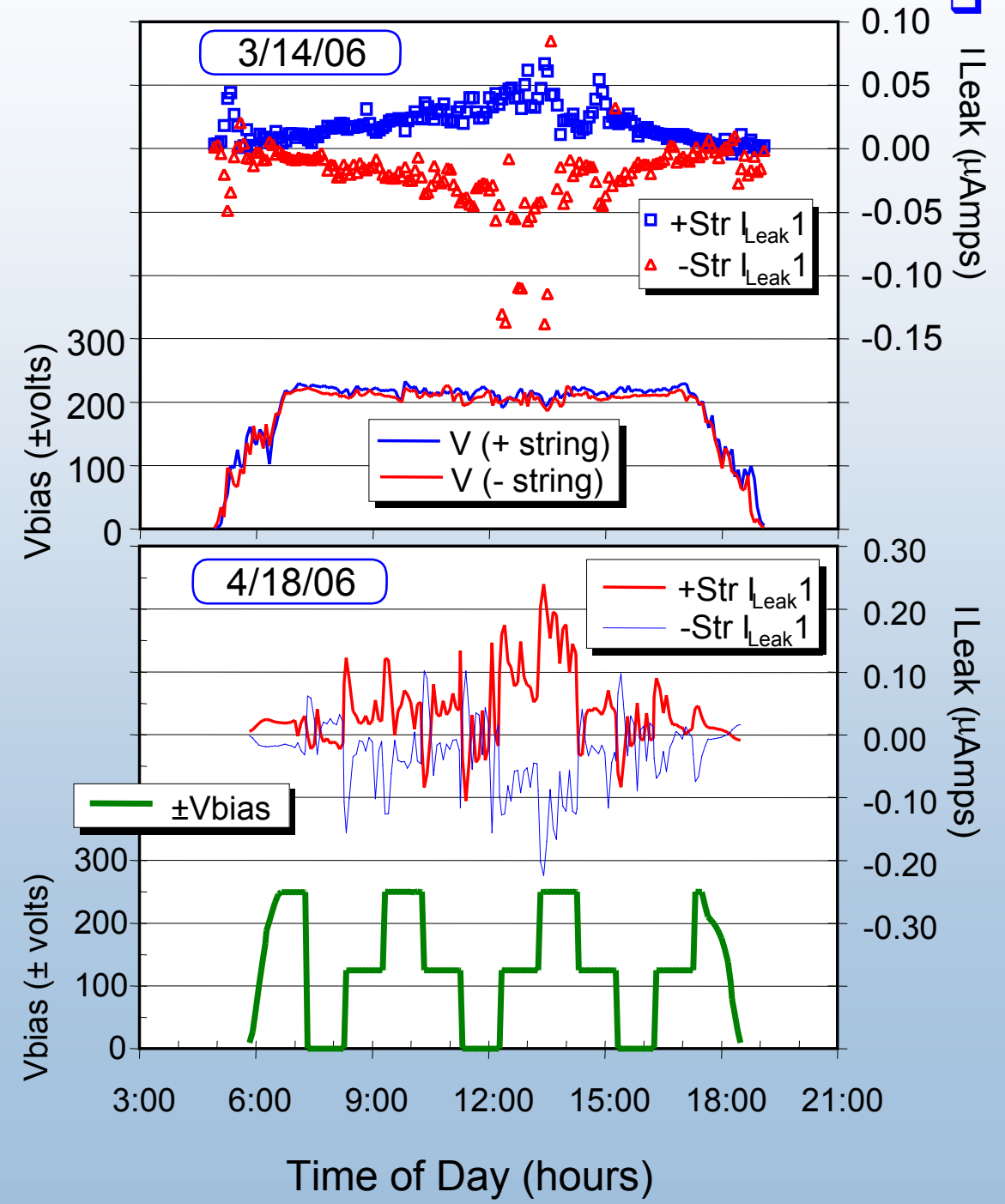

Response of high-voltage leakage currents to bias vs. time of day shown for \pm groups 1 on two days

$>$ Peak-power tracking at top, \& steppedvoltage vs. time profile at bottom,

$>$ Corresponding $\pm \mathrm{V}_{\text {bias }}$ shown, read at left, leakage currents read at right

$>$ For stepped-bias, response appears qualitatively different:

* Forcing effect in phase with large step increments in bias

* Leakage exhibit transients and larger value by $\sim 2 \mathrm{x}$ over peak power

* Currents actually flip polarity for several minutes when decrementing, but not when incrementing

- Ion motion \& relaxation in glass 


\section{Conclusions}

a Performance characterized from both power and energy output considerations:

\begin{tabular}{|c|c|c|c|r|c|}
\hline String & $\begin{array}{c}\text { Average } \\
\text { Air Temp. }\end{array}$ & $\begin{array}{c}\text { Average } \\
\text { Module } \\
\text { Temp. }\end{array}$ & $\begin{array}{c}\text { Irradiance or } \\
\text { Insolation }\end{array}$ & $\begin{array}{r}\text { Eff (\%) } \\
\text { Method }\end{array}$ & PR \\
\hline+ & 14 & 28 & $\begin{array}{r}6.12 \\
\mathrm{~kW}-\mathrm{hrs} / \mathrm{m}^{2} / \mathrm{day}\end{array}$ & $\begin{array}{r}8.12 \\
\text { Energy }\end{array}$ & $84.3 \%$ \\
\hline+ & $\begin{array}{c}\text { Temp. } \\
\text { Corrected }\end{array}$ & 25 & $1001.5 \mathrm{Wm}^{2}$ & $\begin{array}{r}9.11 \\
\text { Power }\end{array}$ & $94.5 \%$ \\
\hline- & 13 & 27 & $\begin{array}{r}6.18 \\
\mathrm{~kW}-\mathrm{hrs} / \mathrm{m}^{2} / \text { day }\end{array}$ & $\begin{array}{r}7.95 \\
\text { Energy }\end{array}$ & $83.5 \%$ \\
\hline- & $\begin{array}{c}\text { Temp. } \\
\text { Corrected }\end{array}$ & 25 & $1001.4 \mathrm{Wm}^{2}$ & $\begin{array}{r}8.87 \\
\text { Power }\end{array}$ & $93.1 \%$ \\
\hline
\end{tabular}

> Significance of energy-based effective efficiency, rows 2, 4:

* Average insolation * effective efficiency (at $13^{\circ}-14^{\circ} \mathrm{C}$ Average daytime air temperature) $=$ delivered energy output per day

- We present energy-based performance temperature coefficient:

- $-0.38 \% /{ }^{\circ} \mathrm{C}$ vs. average daytime air temperatures

a Stability:

$>$ Does not appear to be degrading statistically or otherwise 


\section{Conclusions}

- Leakage Currents:

$>$ Measured and characterized

$>$ Appear thermally activated

$>$ May point to potential corrosion problems in hot environments, where average daytime air temperatures are at, or exceed $25-30{ }^{\circ} \mathrm{C}$ for 200 days or more per year, after 10 years. 


\section{Appendix: IV Trace Statistical}

\section{Filtering for Clear sky vs. Diffuse Illumination}

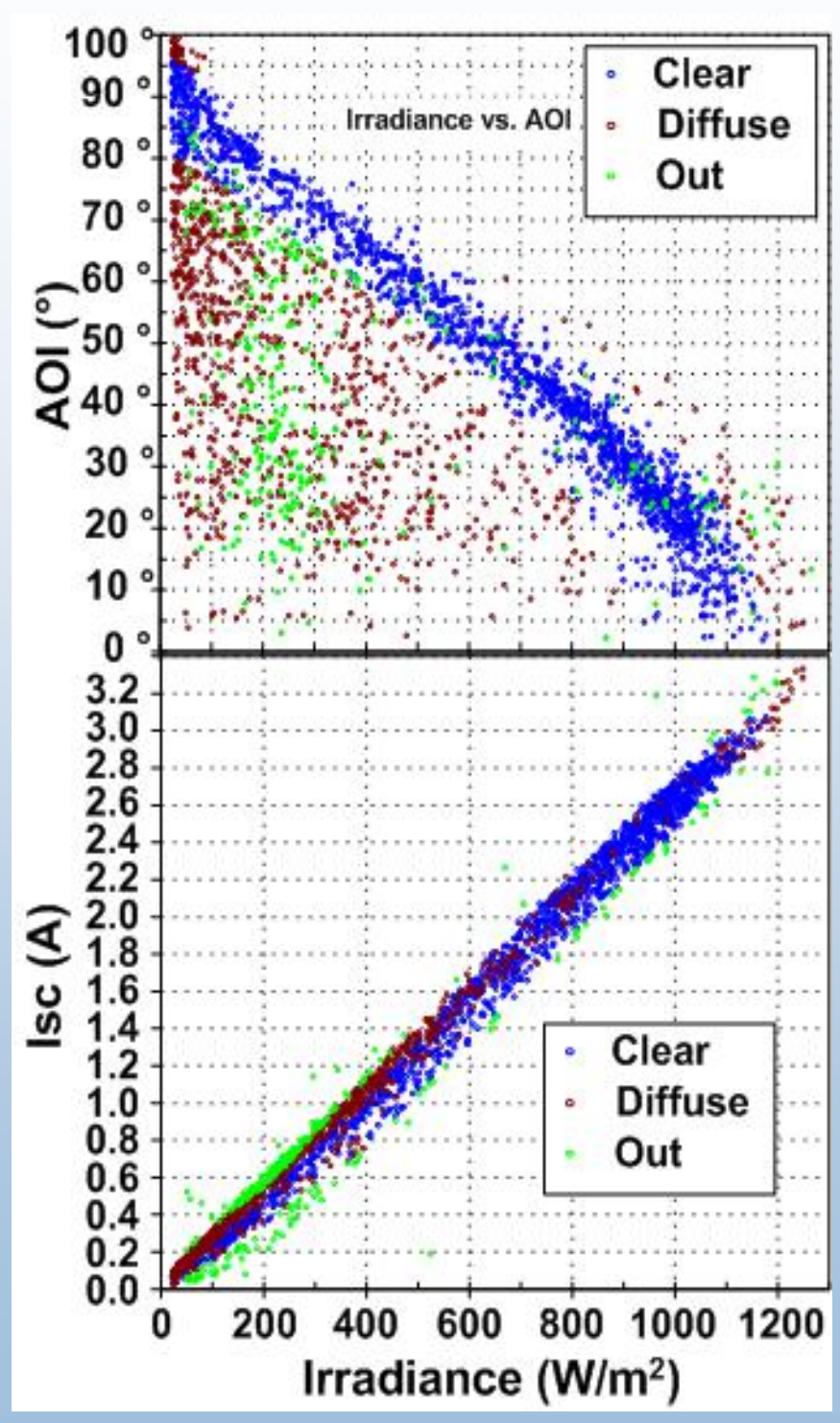

- Two conditions applied: Isc is

linear with Irr \& Irr $=\operatorname{Irr}(\cos (\mathrm{AOI}))$

- $\operatorname{Irr} \sim \mathrm{k}_{2}{ }^{*} \cos (\mathrm{AOI}) \pm 2 \sigma$

$>$ differentiates between predominantly clear vs. diffuse

$>$ Works well, but at larger AOI the diffuse starts to become dominant over direct beam

$\operatorname{Isc} \sim \mathrm{k}_{1} * \operatorname{Irr} \pm 2 \sigma$

$>$ mitigates data traced $\mathrm{w}$. rapid changes in Irr and/or obscuration by snow

$>$ Carried out in piecewise intervals 